\title{
First Untoward Events and Reasons for Tooth Extraction after Nonsurgical Endodontic Treatment in Taiwan
}

\author{
Shib-Chung Chen, DDS, * Ling-Huey Chueh, DDS, MS, ${ }^{+}$Chubsing Kate Hsiao, PhD, ${ }^{\neq}$ \\ Hsin-Ping Wu, MS, ${ }^{\neq}$and Chun-Pin Chiang, DDS, DMSc ${ }^{\mathcal{L}} \|$
}

\section{Abstract}

In this study, 857 teeth having undergone nonsurgical root canal treatment (NSRCT) in Taiwan in 2000 were evaluated during a 5-year follow-up period for first untoward events and reasons for tooth extractions. First untoward events occurred in $83(9.7 \%)$ teeth during this follow-up period, and nonsurgical retreatment was performed for $20(24.1 \%), 4(4.8 \%)$ received apical surgery, and 59 (71.1\%) were extracted. By the end of this 5-year follow-up period a total of 64 (7.5\%) teeth had been extracted. There were 65 reasons for tooth extraction given by the 56 performing dentists who responded to the extraction reason questionnaires; $6(10.7 \%)$ were attributed to endodontically related diseases, $15(26.8 \%)$ to periodontal diseases, 26 $(46.4 \%)$ to large decay or unrestorable tooth, and 18 $(32.1 \%)$ to tooth fracture. The conclusion was that $7.5 \%$ of the NSRCT teeth were extracted by the end of the 5 -year follow-up period, and only $10.7 \%$ of these teeth were extracted as a result of endodontically related diseases. (J Endod 2008;34:671-674)

\section{Key Words}

Endodontic outcome, reasons for tooth extraction, untoward events

From the *Department of Health, The Executive Yuan, Taiwan; ${ }^{\dagger}$ Elite Dental Clinic; ${ }^{\ddagger}$ Department of Public Health, College of Public Health, §School of Dentistry, College of Medicine, and "Dental Department of National Taiwan University Hospital, National Taiwan University; Taipei, Taiwan.

Address requests for reprints to Professor Chun-Pin Chiang, Department of Dentistry, National Taiwan University Hospital, No. 1 Chang-Te Street, Taipei 10048, Taiwan. E-mail address: cpchiang@ntu.edu.tw.

0099-2399/\$0 - see front matter

Copyright (C) 2008 by the American Association of Endodontists.

doi:10.1016/j.joen.2008.03.016
E ndodontic treatment outcome of human teeth can be assessed by a case-controlled or a follow-up epidemiologic study. A case-controlled study evaluates a relatively small number of endodontic treatment and control cases. Because the criteria used for assessing success and failure might be different, the endodontic treatment outcomes vary considerably among several studies (1). The follow-up epidemiologic study examines a very large number of endodontically treated cases from a general population. There are 3 examples of this type of epidemiologic study that uses an alternative approach to evaluate the nonsurgical root canal treatment (NSRCT) cases from the database of a nationwide health insurance organization (2-4). Because an epidemiologic study assesses a large number of endodontic treatments performed by both general practitioners and endodontists, its yielded success rate might represent outcomes in the general population (2-4).

In Taiwan, a national healthcare plan was implemented in March 1995. This plan covers nearly all medical treatments and a portion of the dental treatments including surgical and nonsurgical endodontic procedures. The National Health Insurance (NHI) is managed by the Bureau of $\mathrm{NHI}(\mathrm{BNHI})$, which insures approximately 21 million individuals ( $95.5 \%$ of the total population of 22 million). Because the providers must make a claim for each treatment to the BNHI, a large computerized medical and dental database for each insured individual has been kept since March 1995. Access to BNHI's invaluable national data set makes it possible to study the first untoward events including nonsurgical retreatment, apical surgery, and tooth extraction as well as tooth retention rate after NSRCT nationwide.

Two large epidemiologic studies with a long follow-up period reported very high tooth retention rates $(94 \%-97 \%)$ from assessment of a large number of NSRCT cases collected from an insurance company database $(2,3)$. These results stimulated our group to study the first untoward events and tooth retention rate during a 5-year follow-up period for 1,557,547 teeth receiving NSRCT in Taiwan in 1998. We found that first untoward events occurred in 159,680 (10.3\%) NSRCT teeth during the 5-year follow-up period. Moreover, a high 5 -year tooth retention rate of $92.9 \%$ was discovered (4). In another previous study, we evaluated the technical quality of root canal filling in a total of 1085 NSRCT cases randomly selected from a large representative sample of the Taiwanese population in 2000 . We found that only $30.3 \%$ of NSRCT teeth had good quality endodontic work (GQEW), and the remaining $69.7 \%$ of NSRCT teeth had modest quality endodontic work (MQEW) (5). Because the records of the technical quality of root canal fillings in these 1085 NSRCT teeth were available, we were able to assess the first untoward events and tooth retention rate during a 5 -year follow-up period of these teeth. In addition, we compared the incidence of each untoward event, the tooth extraction rate, and the number of teeth extracted each year between teeth with GQEW and teeth with MQEW. We also tried to identify the causes for tooth extraction after NSRCT by sending questionnaires to the dentists who performed the extraction procedure.

\section{Materials and Methods}

The information of each NSRCT tooth was collected from the database of NHI in Taiwan. Each NSRCT tooth was followed for a continuous period of 5 years from the completion date of its endodontic procedure in 2000. The subsequent treatment codes and dates for each NSRCT tooth within the 5-year follow-up period were collected. Of particular interest was the evaluation of the first and the last untoward events, which were defined as nonsurgical retreatment, apical surgery, and extraction in 2 prior 
TABLE 1. Incidences of First Untoward Events in Teeth with GQEW and in Teeth with MQEW During 5-year Follow-up Period

\begin{tabular}{ccccc}
\hline \multirow{2}{*}{ All Teeth } & \multicolumn{3}{c}{ First Untoward Events, No. of Cases (\%) } \\
\cline { 2 - 5 } & $\begin{array}{c}\text { Nonsurgical } \\
\text { Retreatment }\end{array}$ & $\begin{array}{c}\text { Apical } \\
\text { Surgery }\end{array}$ & $\begin{array}{c}\text { Tooth } \\
\text { Extraction }\end{array}$ & Total \\
\hline $\begin{array}{c}\text { Teeth with GQEW } \\
(\mathrm{n}=220)\end{array}$ & $1(0.5)$ & $2(0.9)$ & $17(7.7)$ & $20(9.1)$ \\
$\begin{array}{c}\text { Teeth with MQEW } \\
(\mathrm{n}=637)\end{array}$ & $19(3.0)$ & $2(0.3)$ & $42(6.6)$ & $63(9.9)$ \\
$\begin{array}{c}\text { Total follow-up } \\
\text { teeth }(\mathrm{n}=857)\end{array}$ & $20(2.3)$ & $4(0.5)$ & $59(6.9)$ & $83(9.7)$ \\
\hline
\end{tabular}

GQEW, good quality endodontic work; MQEW, modest quality endodontic work.

epidemiologic studies $(2,3)$. The occurrence of untoward events was analyzed by searching the database for specific treatment codes that were then used to identify the specific treatment modality, which included nonsurgical retreatment, apicoectomy, root-end filling, and simple or complicated tooth extraction.

The first subsequent procedure for each NSRCT tooth was analyzed to disclose whether any untoward event occurred to each tooth after the completion of NSRCT. In addition, the total annual number and incidence of each untoward event for all NSRCT teeth were calculated. The last related procedure for each NSRCT tooth was used to calculate how many teeth were extracted and in turn to determine the percentage of teeth retained after completion of the full 5-year follow-up period. In this study, the patients' outcome criteria were applied blindly because the dentists submitting their claims were not aware that the data would be used for future quality evaluation and outcome analysis.

This study used both an adequate filling length and a complete obturation in the apical one third of the root canal as the criteria for defining a GQEW as previously described (5). These criteria are advocated by the European Society of Endodontology (1994), ie, a radiographically dense filling with its end located between 0 and $2 \mathrm{~mm}$ from the apex. However, a gutta-percha-filled root canal with either an inadequate filling length or an incomplete obturation in the apical one third of the root canal was defined as an MQEW.

To identify the causes for tooth extraction, a questionnaire was sent to the dentists who performed the extraction procedure. The dentists were asked to fill in the causes for tooth extraction including endodontically related diseases, periodontal diseases, large decay or unrestorable tooth, and tooth fracture. Multiple causes for tooth extraction were allowed.

The $\chi^{2}$ test was used to compare the incidence of each untoward event, the tooth extraction rate, and the annual number of extracted teeth between teeth with GQEW and teeth with MQEW. A $P$ value of less than .05 was considered statistically significant for all tests.

\section{Results}

\section{Incidences of First Untoward Events During 5-Year Follow-Up} Period

Among the 1085 teeth in which the technical quality of NSRCT was known, we were able to trace the dental treatment records of the 857 teeth by identification number and specific treatment codes in the database of the Taiwan Department of Health. Of these 857 teeth, 220 had GQEW, and 637 had MQEW. The first untoward events were found in 83 teeth $(9.7 \%)$ during the 5-year follow-up period. Tooth extraction was the most common first untoward event $(71.1 \%)$ followed by the nonsurgical retreatment $(24.1 \%)$ and apical surgery (4.8\%). There was no significant difference in the incidences of the first untoward events between the teeth with GQEW and the teeth with MQEW $(P=.343$, Table 1$)$.

\section{Distribution of 83 First Untoward Events in Each of the 5 Follow-Up Years}

The distribution of 83 first untoward events in each of the 5 follow-up years is shown in Table 2. There were more untoward events (25 cases, 30.1\%) during the first follow-up year and less untoward events (12 cases, $14.5 \%$ ) during the second follow-up year compared with the number of untoward events during the next 3 years $(P=.068$, marginal significance).

\section{Tooth Extraction Rates for Anterior Teeth, Premolars, and Molars During 5-Year Follow-Up Period}

Sixty-four (7.5\%) of 857 follow-up teeth were extracted after completion of the 5-year follow-up period, yielding a 5-year tooth retention rate of $92.5 \%$. Of these 64 extracted teeth, 18 had a GQEW, and 46 had an MQEW; moreover, 59 were extracted at first untoward events, 3 after nonsurgical retreatment, and 2 after apical surgery. In addition, more molars (46.9\%) were extracted than premolars (29.7\%) and anterior teeth $(23.4 \%)$. There was no significant difference in tooth extraction rates for anterior teeth, premolars, and molars between the teeth with GQEW and the teeth with MQEW $(P=.698$, Table 3).

\section{Distribution of 64 Extracted Teeth in Each of the 5 Follow-Up Years}

The distribution of 64 extracted teeth in each of the 5 follow-up years is shown in Table 4. Although there were more teeth (16 teeth, $25.0 \%)$ extracted during the first year of follow-up and less teeth $(9$ teeth, $14.1 \%$ ) extracted during the second year of follow-up compared with the number of teeth extracted during the next 3 years, there was no significant difference in the annual number of extracted teeth between the teeth with GQEW and the teeth with MQEW $(P=.330$, Table 4).

\section{Reasons for Tooth Extraction After NSRCT}

In this study, 64 NSRCT teeth were extracted by 64 different dentists. Questionnaires were sent to the 64 dentists who performed the tooth extractions; 56 gave a response. Of these 56 dentists, 47 gave 1 reason and 9 gave 2 reasons for the tooth extraction. Of these 65 reasons for tooth extraction, 6 (10.7\%) were attributed to endodontically related diseases, 15 (26.8\%) to periodontal diseases, 26 (46.4\%) to large decay or unrestorable tooth, and 18 (32.1\%) to tooth fracture (Table 5).

\section{Discussion}

Our previous study and this study assessed the first untoward events and tooth retention during a 5-year follow-up period for

TABLE 2. Distribution of 83 First Untoward Events in Each of the 5 Follow-up Years

\begin{tabular}{lcccrrr}
\hline \multirow{2}{*}{ Regions } & \multicolumn{5}{c}{ No. of Cases (\%) } \\
\cline { 2 - 5 } & First Year & Second Year & Third Year & Fourth Year & Fifth Year & \multicolumn{1}{c}{ Total } \\
\hline Nonsurgical retreatment & $6(30.0)$ & $5(25.0)$ & $4(20.0)$ & $1(5.0)$ & $4(20.0)$ & $20(100.0)$ \\
Apical surgery & $4(100.0)$ & $0(0.0)$ & $0(0.0)$ & $0(0.0)$ & $0(0.0)$ & $4(100.0)$ \\
Tooth extraction & $15(25.4)$ & $7(11.9)$ & $11(18.6)$ & $14(23.7)$ & $12(20.3)$ & $59(100.0)$ \\
Total & $25(30.1)$ & $12(14.5)$ & $15(18.1)$ & $15(18.1)$ & $16(19.3)$ & $83(100.0)$ \\
\hline
\end{tabular}


TABLE 3. Tooth Extraction Rates for Anterior Teeth, Premolars, and Molars in Teeth with GQEW and in Teeth with MQEW During 5-year Follow-up Period

\begin{tabular}{lccccc}
\hline & \multicolumn{5}{c}{ No. of Cases (\%) } \\
\cline { 2 - 5 } & $\begin{array}{c}\text { All } \\
\text { Teeth }\end{array}$ & $\begin{array}{c}\text { Anterior } \\
\text { Teeth }\end{array}$ & Premolars Molars & $\chi^{2}$ Test \\
\hline $\begin{array}{c}\text { Teeth with } \\
\text { GQEW } \\
(n=220)\end{array}$ & $18(8.2)$ & $3(1.4)$ & $8(3.6)$ & $7(3.2)$ & $P=.698$ \\
$\begin{array}{c}\text { Teeth with } \\
\text { MQEW } \\
(n=637)\end{array}$ & $46(7.2)$ & $12(1.9)$ & $11(1.7)$ & $23(3.6)$ \\
$\begin{array}{c}\text { Total follow-up } \\
\text { teeth } \\
(n=857)\end{array}$ & $64(7.5)$ & $15(1.8)$ & $19(2.2)$ & $30(3.5)$ \\
\hline
\end{tabular}

GQEW, good quality endodontic work; MQEW, modest quality endodontic work.

1,557,547 and 857 NSRCT teeth in Taiwan in 1998 and 2000, respectively (4). Although the sample sizes of the 2 studies were different, the 5 -year tooth retention rate obtained in this study (92.5\%) was very close to that $(92.9 \%)$ reported in our previous study (4). Because the 857 NSRCT teeth we examined in the present study were randomly selected from 1,588,217 NSRCT cases representative of a large sample from the Taiwan population, we suggest that the results obtained in this study might relatively represent the results obtained from a general population in Taiwan.

Recently, an epidemiologic model with a very large sample size was used to evaluate endodontic treatment outcome $(2,3)$; in this type of study, tooth retention became an evaluative criterion for clinical outcome after NSRCT. Three recent studies have evaluated tens of thousands or more than one million NSRCT cases. Lazarski et al (2) reported that $94.4 \%$ of NSRCT teeth remained functional during an average follow-up period of 3.5 years. Salehrabi and Rotstein (3) found that $97 \%$ of teeth were retained in the oral cavity 8 years after initial NSRCT. Our recent study showed that $92.9 \%$ of $1,557,547$ teeth treated in Taiwan in 1998 were retained in the oral cavity 5 years after an NSRCT (4). This study also demonstrated a 5-year tooth retention rate of $92.5 \%$. Other follow-up investigations reviewed by Friedman and Mor (1) have also shown that $91 \%-97 \%$ of endodontically treated teeth remained functional over time. The results of the above studies indicated a high tooth retention incidence of greater than $91 \%$ after NSRCT. It is reasonable to conclude that NSRCT is a valuable dental procedure because of the high tooth retention rate.

Epidemiologic studies also provide some information on the relatively small group of teeth experiencing an untoward event after initial NSRCT. The present study found that $71.1 \%$ of the 83 teeth with the first untoward event were extracted during the 5 -year follow-up period. This result was comparable to the corresponding result $(65.5 \%)$ of our previous study (4). Lazarski et al (2) reported that $59 \%$ of 4,212 teeth suffering a first untoward event were extracted during a minimum follow-up interval of 2 years. Salehrabi and Rotstein (3) also demonstrated that $73.5 \%$ of 57,361 teeth with the first untoward events were extracted.
All 4 studies indicated that tooth extraction was the most common initial untoward event after NSRCT.

The frequencies of apical surgery after NSRCT were 15\% and $16.4 \%$ in the studies of Lazarski et al (2) and Salehrabi and Rotstein (3), respectively, with both rates higher than the $4.8 \%$ and $2.8 \%$ demonstrated in this study and in our previous study, respectively (4). In the USA, endodontics is one of the American Dental Association's recognized specialties. Lazarski et al found that approximately $70 \%$ of all apical surgeries after NSRCT are performed by endodontists in the USA. In Taiwan, apical surgery is considered a high-level technique always taught in postgraduate courses; therefore, it is performed mostly by endodontists or oral surgeons. Thus, global differences in endodontic training might contribute to differences in rates of apical surgery versus extraction. In the present study, the nonsurgical-retreatment frequency was $24.1 \%$, which was comparable to that (26\%) determined by Lazarski et al (2), but it was slightly higher than that (10.1\%) reported by Salehrabi and Rotstein (3) and slightly lower than that (31.7\%) demonstrated in our previous study (4).

In the present study, it was surprising to find that there was no significant difference in the tooth extraction rate for anterior teeth, premolars, and molars between the teeth with GQEW and the teeth with MQEW. Furthermore, the overall tooth extraction rate was slightly higher in the group of teeth with GQEW (8.2\%) than in the group of teeth with MQEW (7.2\%), although the difference was not significant. This finding stimulated us to further investigate the causes for tooth extraction in our samples. We discovered that only $10.7 \%$ of NSRCT teeth were extracted as a result of endodontically related diseases, and $89.3 \%$ of NSRCT teeth were extracted as a result of nonendodontically related diseases such as periodontal diseases $(26.8 \%)$, large decay or unrestorable tooth $(46.4 \%)$, and tooth fracture $(32.1 \%)$. Comparable findings of reasons for tooth extraction after NSRCT were also reported by others. Vire (6) showed that tooth extractions after NSRCT procedures were due to prosthetic failure in $59.4 \%$ of cases, periodontal failure in $32 \%$ of cases, and endodontic failure in $8.6 \%$ of cases. Fuss et al (7) reported that the 3 major reasons for extraction of endodontically treated teeth were unrestorable teeth $(43.5 \%)$, endodontic failure $(21.1 \%)$, and vertical root fracture (10.9\%). Lazarski et al (2) also demonstrated a significant influence of subsequent restoration and build-up on the tooth extraction after NSRCT. The above-mentioned findings indicate that only a relatively small percentage $(8.6 \%-$ $21.1 \%)$ of NSRCT teeth are extracted as a result of endodontic failure. There is an inevitable need of doing prosthodontic restoration on teeth after NSRCT to prevent the teeth from large decay and tooth fracture. Moreover, periodontal consultation before NSRCT is necessary when the teeth have moderate or high severity of periodontal diseases.

In conclusion, tooth extraction is the most common first untoward event after NSRCT. Only $10.7 \%$ of NSRCT teeth were extracted as a result of endodontically related diseases. There was a high tooth retention rate of $92.5 \%$ five years after NSRCTs in Taiwan.

TABLE 4. Distribution of 64 Extracted Teeth in Teeth with GQEW and in Teeth with MQEW During 5-year Follow-up Period

\begin{tabular}{|c|c|c|c|c|c|c|}
\hline \multirow{2}{*}{ Regions } & \multicolumn{6}{|c|}{ No. of Cases (\%) } \\
\hline & First Year & Second Year & Third Year & Fourth Year & Fifth Year & Total \\
\hline Teeth with GQEW $(n=220)$ & $6(33.3)$ & $3(16.7)$ & $3(16.7)$ & $1(5.5)$ & $5(27.8)$ & $18(100.0)$ \\
\hline Teeth with MQEW $(n=637)$ & $10(27.8)$ & $6(16.7)$ & $9(25.0)$ & $13(36.1)$ & $8(22.2)$ & $36(100.0)$ \\
\hline Total follow-up teeth $(n=857)$ & $16(25.0)$ & $9(14.1)$ & $12(18.8)$ & $14(21.9)$ & $13(20.3)$ & $64(100.0)$ \\
\hline
\end{tabular}

GQEW, good quality endodontic work; MQEW, modest quality endodontic work. 
TABLE 5. A Total of 65 Reasons for Tooth Extraction after NSRCT Given by 56 Responding Dentists Including 47 Giving 1 Reason and 9 Giving 2 Reasons for Tooth Extraction

\begin{tabular}{|c|c|c|c|c|}
\hline \multirow[b]{2}{*}{ Reasons For Tooth Extraction } & \multicolumn{4}{|c|}{ Reasons For Tooth Extraction } \\
\hline & $\begin{array}{l}\text { Endodontically } \\
\text { Related Diseases }\end{array}$ & $\begin{array}{l}\text { Periodontal } \\
\text { Diseases }\end{array}$ & $\begin{array}{l}\text { Large Decay Or } \\
\text { Unrestorable Tooth }\end{array}$ & Tooth Fracture \\
\hline One reason $(n=47)$ & 2 & 11 & 20 & 14 \\
\hline \multicolumn{5}{|l|}{ Two reasons $(n=9)$} \\
\hline Endodontically related diseases & - & 1 & 2 & 1 \\
\hline Periodontal diseases & 1 & - & 2 & 1 \\
\hline Large decay or unrestorable tooth & 2 & 2 & - & 2 \\
\hline Tooth fracture & 1 & 1 & 2 & - \\
\hline Total reasons $(n=65)$ & 6 & 15 & 26 & 18 \\
\hline
\end{tabular}

NSRCT, nonsurgical root canal treatment.

\section{Acknowledgments}

The authors thank Sister Mary Ellen Kerrigan for editing the manuscript and the Bureau of National Heath Insurance in Taiwan for the help provided during the study. This research was supported by grant DOH95-HI-1001 from the Department of Health, the Executive Yuan, Taiwan.

\section{References}

1. Friedman S, Mor C. The success of endodontic therapy: healing and functionality J Calif Dent Assoc 2004;32:493-503.
2. Lazarski MP, Walker WA, Flores CM, Schindler WG, Hargreaves KM. Epidemiological evaluation of the outcomes of nonsurgical root canal treatment in a large cohort of insured dental patients. J Endod 2001;27:791-6.

3. Salehrabi R, Rotstein I. Endodontic treatment outcomes in a large patient population in the USA: an epidemiological study. J Endod 2004;30:846-50.

4. Chen SC, Chueh LH, Hsiao CK, Tsai MY, Ho SC, Chiang CP. An epidemiological study of tooth retention after nonsurgical endodontic treatment in a large population in Taiwan. J Endod 2007;33:226-9.

5. Chueh LH, Chen SC, Lee CM, et al. Technical quality of root canal treatment in Taiwan. Int Endod J 2003;36:416-22.

6. Vire DE. Failure of endodontically treated teeth: classification and evaluation. J Endod 1991;17:338-42.

7. Fuss Z, Lustig J, Tamse A. Prevalence of vertical root fractures in extracted endodontically treated teeth. Int Endod J 1999;32:283-6. 